\title{
The envelope attractor of non-strict multivalued dynamical systems with application to the 3D Navier-Stokes and reaction-diffusion equations
}

\author{
P.E. Kloeden ${ }^{1}$, P. Marín-Rubio ${ }^{2}$ and J. Valero ${ }^{3}$ \\ ${ }^{1}$ Institut für Mathematik, Goethe Universität, D-60054 Frankfurt am Main, Germany \\ 2 Departamento de Ecuaciones Diferenciales y Análisis Numérico, \\ Universidad de Sevilla, Apdo. de Correos 1160, 41080-Sevilla, Spain \\ ${ }^{3}$ Universidad Miguel Hernandez de Elche, Centro de Investigación Operativa, \\ Avda. Universidad s/n, 03202-Elche, Spain \\ E-mails: kloeden@math.uni-frankfurt.de; pmr@us.es; jvalero@umh.es
}

\begin{abstract}
Multivalued semiflows generated by evolution equations without uniqueness sometimes satisfy a semigroup set inclusion rather than equality because, for example, the concatentation of solutions satisfying an energy inequality almost everywhere may not satisfy the energy inequality at the joining time. Such multivalued semiflows are said to be non-strict and their attractors need only be negatively semi-invariant. In this paper the problem of enveloping a non-strict multivalued dynamical system in a strict one is analyzed and their attactors are compared. Two constructions are proposed. In the first, the attainability set mapping is extending successively to be strict at the dyadic numbers, which essentially means (in the case of the Navier-Stokes system) that the energy inequality is satisfied piecewise on successively finer dyadic subintervals. The other deals directly with trajectories and their concatenations, which are then used to define a strict multivalued dynamical system. The first is shown to be applicable to the three-dimensional Navier-Stokes equations and the second to a reaction-diffusion problem without unique solutions.
\end{abstract}

AMS Subject Classification: 35B40, 35B41, 35K55, 35K57, 35Q30, 37B25, 58C06.

Key words: Multivalued dynamical systems, non-strict multivalued semiflows, non-strict and strict global attractors, 3D Navier-Stokes equations, reaction-diffusion equations

\section{Introduction}

Differential equations without uniqueness and differential inclusions generate multivalued semigroups under appropriate conditions ensuring the forwards existence of solutions, see, e.g., the classical references, Roxin [17] and Szegö and Treccani [19]. The situation in some particular partial differential models in applied sciences, such as the three-dimensional Navier-Stokes equations, is more complicated, since there are different kinds of solutions that one can consider. With the Navier-Stokes equations one usually restricts to weak solutions satisfying an energy inequality (it is, in fact, not known if there are weak solutions that do not satisfy such an inequality), but such inequalities are usually satisfied only "almost 
surely", so the concatenation of two weak solutions need not satisfy the energy inequality since it may not be valid at the instant where they are to be joined. This leads to a semigroup property with an inclusion $\Phi(t+s, x) \subset \Phi(t, \Phi(s, x))$ rather than with a strict equality $\Phi(t+s, x)=\Phi(t, \Phi(s, x))$ and a resulting attractor $\mathcal{A}$ is only negatively semi-invariant, i.e., with $\mathcal{A} \subset \Phi(t, \mathcal{A})$, rather than strictly invariant, i.e., $\mathcal{A}=\Phi(t, \mathcal{A})$, so the property of being positively semi-invariant fails. A similar situation appears for reaction-diffusion equations when we consider solutions which are regular in every interval of time of the type $[\varepsilon, T]$ with $\varepsilon>0$. The theory of such non-strict multivalued semiflows and their attractors is developed in the paper Melnik and Valero [14], see also the monographs Kapustyan et al [9], Zgurovsky et al $[22]$ and $[10,12,15]$.

The aim of this paper is to construct an "envelope" of a given non-strict multivalued semiflow, that is, a strict multivalued semiflow containing the original non-strict multivalued semiflow. We give two main constructions to reach our goal.

In the first one we consider a general multivalued non-strict semiflow $\Phi$. The envelope will be built up successively concatenating the images of $\Phi$ in a piecewise manner with switches at dyadic time instants $\widehat{\mathfrak{D}}_{n}:=\left\{j 2^{-n}: j=0,1,2,3, \cdots\right\}$ for $n=1,2, \cdots$. We then interpret the classical attractor of this envelope system as an enveloping attractor of the original system under all possible switching. There are, however, technical difficulties that prevent us from realising our ultimate aim and we are forced to make a number of modifications and compromises. The reasons for this are, nevertheless, interesting and our results provide useful insights into the dynamics of non-strict multivalued semiflows. In particular, we obtain first an envelope semiflow $\Phi^{(\infty)}$ which is strict at the dyadic points. However, this map has not closed values in general, and then a global attractor cannot be obtained (the property of negatively semi-invariance fails now). Taking a kind of closure of this map $\Phi^{(\infty)}$ we define a new non-strict semiflow $\bar{\Phi}^{(\infty)}$. But, since it is close to a strict one, we can prove that its global attractor is weakly positively invariant in the sense that for any initial data in the attractor there exists a $\bar{\Phi}^{(\infty)}$-trajectory lying in the attractor for all positive times.

These results are suitable for the application to the 3D Navier-Stokes system. Roughly speaking, in this application the envelope semiflow is constructed by concatenating weak solutions that satisfy the energy inequality almost surely.

In the second construction we consider a semiflow generated by some set of functions satisfying the translation property. By using a special type of concatenations for such functions we define a new set of functions which satisfy not only the translation property, but the concatenation one as well. Hence, this new set of functions generates a strict multivalued semiflow. Moreover, using an extra assumption on this set we can prove that the semiflow possesses an invariant global attractor.

We apply this abstract theory to a multivalued semiflow generated by the regular solutions of a reaction-diffusion equation. Unfortunately, we could not apply it to the Navier-Stokes system.

The paper is structured as follows. In Section 2 we recall some basic properties of multivalued autonomous dynamical systems generated by multivalued semiflows (often called m-semiflows for short) and attractors, emphasizing the different invariance properties of the attractor depending on whether the m-semiflow is strict or not.

In Section 3 we introduce the idea of an envelope system coming from an abstract m-semiflow. This construction is done step by step in several subsections, where we analyze the different properties of the constructed objects that lead, in the end, to the envelope system and the corresponding envelope attractor. The main result of this section shows the weak positive semi-invariance of the attractor.

In Section 4 the second approach is carried out. In this way, it is possible to obtain a strict envelope with an invariant attractor. 
Finally, Section 5 is devoted to illustrate the applicability of our results in the two examples commented above, the three-dimensional Navier-Stokes equations and a reaction-diffusion problem, which generate non-strict semiflows. For the Navier-Stokes system we apply the first type of results, those given in Section 3 , obtaining a weakly positively semi-invariant attractor. The reaction-diffusion problem can be handled within the framework developed in Section 4, leading to a strict invariant attractor.

\section{Multivalued dynamical systems}

Let $X$ be a complete metric space with metric $\rho$ and $\mathbb{T}$ be a nontrivial subgroup of the additive group of the real numbers $\mathbb{R}, \mathbb{T}^{+}:=\mathbb{T} \cap \mathbb{R}^{+}$Denote by $\mathcal{P}(X)$ the set of all nonempty subsets of $X$ and by $\mathcal{C}(X)$ the set of all nonempty closed subsets. We say that a multi-valued map $\Phi: \mathbb{T}^{+} \times X \rightarrow \mathcal{P}(X)$ is a multivalued semi-flow if the following conditions are satisfied:

1. $\Phi(0, x)=\{x\} \quad$ for all $x \in X$,

2. $\Phi\left(t_{1}+t_{2}, x\right) \subset \Phi\left(t_{1}, \Phi\left(t_{2}, x\right)\right) \quad$ for all $t_{1}, t_{2} \in \mathbb{T}^{+}$and $x \in X$,

where $\Phi(t, B)=\cup_{x \in B} \Phi(t, x)$ for $B \subset X$. The multivalued semiflow is called strict if $\Phi\left(t_{1}+t_{2}, x\right)=$ $\Phi\left(t_{1}, \Phi\left(t_{2}, x\right)\right)$ for all $t_{1}, t_{2} \in \mathbb{T}^{+}$and $x \in X$.

We shall also say that the map $\Phi$ generates (or simply is) a multivalued dynamical system. Its asymptotic behaviour is typically characterized by a global attractor.

Definition 1 A set $\mathcal{A} \subset X$ is called a global attractor for $\Phi$ if:

1. it is negatively semi-invariant, i.e., $\mathcal{A} \subset \Phi(t, \mathcal{A})$ for all $t \in \mathbb{T}^{+}$;

2. it is attracting, i.e.,

$$
\operatorname{dist}_{X}(\Phi(t, B), \mathcal{A}) \rightarrow 0 \quad \text { as } t \rightarrow+\infty,
$$

for all bounded subsets $B$ of $X$.

Here

$$
\operatorname{dist}_{X}(C, A):=\sup _{c \in C} \operatorname{dist}_{X}(c, A) \quad \text { with } \quad \operatorname{dist}_{X}(c, A):=\inf _{a \in A}\|c-a\|
$$

is the Hausdorff semi-distance.

We note that, if the global attractor is bounded, then the global attractor $\mathcal{A}$ is minimal, i.e., for any set $Y$ satisfying (1) we have $\mathcal{A} \subset \bar{Y}$. In many applications the global attractor often has additional properties such as compactness or strict invariance. In particular, we say that $\mathcal{A}$ is invariant if $\mathcal{A}=$ $\Phi(t, \mathcal{A})$ for all $t \geq 0$.

Definition 2 The multivalued map $x \rightarrow \Phi(t, x)$ is said to be upper semi-continuous if for any neighborhood $\mathcal{N}$ of $\Phi(t, x)$ there exists $\delta>0$ such that $\Phi(t, y) \subset \mathcal{N}$ when $\rho(y, x)<\delta$.

If $\Phi$ is upper semi-continuous, then for any $\varepsilon>0$ there exists $\delta>0$ such that

$$
\operatorname{dist}_{X}(\Phi(t, x), \Phi(t, y))<\varepsilon \quad \text { if } \rho(x, y)<\delta .
$$

The converse is true if $\Phi$ has compact values.

The following result was proved in [14, Theorem 4 and Remark 8]: 
Theorem 3 Let $\Phi$ be a multivalued semiflow such that $\Phi(t, \cdot): X \rightarrow \mathcal{C}(X)$ is upper semi-continuous for each $t \in \mathbb{T}^{+}$. If there exists a compact subset $K$ of $X$ such that

$$
\operatorname{dist}_{X}(\Phi(t, B), K) \rightarrow 0 \quad \text { as } t \rightarrow \infty
$$

for any bounded subset $B \subset X$, then $\Phi$ has the global compact attractor $\mathcal{A}$, which is the minimal closed set attracting each bounded set $B \subset X$. Moreover,

$$
\mathcal{A}=\omega(K)=\bigcap_{t \geq 0} \overline{\bigcup_{\tau \geq t} \Phi(\tau, K)} \subset K
$$

and, if $\Phi$ is strict, then $\mathcal{A}$ is invariant.

Remark 4 The statement remains valid if the map $\Phi(t, \cdot)$ is not upper semi-continuous, but has a closed graph (see [15]).

The set $\omega(K)$ is called the omega-limit set of $K$ in the literature.

It is clear that, if $\Phi$ has an absorbing bounded set and if the map $\Phi(t, \cdot)$ is compact for some $t>0$, then a compact set $K$ satisfying (2) exists.

\section{Definition of the envelope systems}

We now consider the continuous time case $\mathbb{T}=\mathbb{R}$ and restrict attention, without loss of generality, to the unit interval $[0,1]$. Denote the $n$-dyadic fractions

$$
\mathfrak{D}_{n}:=\left\{j 2^{-n}: j=0, \cdots, 2^{n}\right\}
$$

and note that $\mathfrak{D}_{n} \subset \mathfrak{D}_{n+1}$ for each $n \in \mathbb{N}$.

Let $\Phi$ be a (not necessarily strict) m-semiflow. Define $\Phi^{(0)}(t, x) \equiv \Phi(t, x)$ for all $x \in X$ and $t \in[0,1]$. Then for $n=1,2, \cdots$ define $\Phi^{(n)}(t, x)$ by

$$
\Phi^{(n)}(t, x)=\Phi\left(t-j 2^{-n}, \Phi^{(n-1)}\left(j 2^{-n}, x\right)\right)
$$

for $t \in\left[j 2^{-n},(j+1) 2^{-n}\right]$ with $j=0, \cdots, 2^{n}-1$ and each $x \in X$. Thus, $\Phi^{(n)}(t, x) \subset \Phi^{(n+1)}(t, x)$ for all $t \in[0,1]$.

It is clear by the construction that, for each each $n=1,2, \cdots$,

$$
\Phi^{(n)}(t+s, x) \subset \Phi^{(n)}\left(s, \Phi^{(n)}(t, x)\right)
$$

for all $s, t \in[0,1]$ with $s+t \in[0,1]$, in general, while

$$
\Phi^{(n)}\left(t_{j}+s_{j}, x\right)=\Phi^{(n)}\left(s_{j}, \Phi^{(n)}\left(t_{j}, x\right)\right)
$$

for all $s_{j}, t_{j} \in \mathfrak{D}_{n}$ with $s_{j}+t_{j} \in \mathfrak{D}_{n}$.

We make the additional assumptions:

Assumption A1: $\Phi(t, x)$ is compact for all $t \geq 0$ and $x \in X$. 
Assumption A2: $x \mapsto \Phi(t, x)$ is upper semi-continuous in $x$ for each $t$ in $\mathbb{R}^{+}$.

Note that if Assumption A1 holds, then Assumption A2 is equivalent to

$$
\operatorname{dist}_{X}\left(\Phi(t, x), \Phi\left(t, x^{*}\right)\right) \rightarrow 0 \text { as } x \rightarrow x^{*} .
$$

If $K$ is compact in $X$, then $\Phi(t, K)$ is compact by Assumptions A1 and A2 due to a lemma of Roxin [17]. Moreover, $\Phi\left(t, K_{j}\right) \rightarrow \Phi(t, K)$ in the upper semi-continuous sense for compact sets such that $K_{j}$ $\rightarrow K$ in $X$, i.e.,

$$
\operatorname{dist}_{X}\left(\Phi\left(t, K_{j}\right), \Phi(t, K)\right) \rightarrow 0 \quad \text { as } \quad \operatorname{dist}_{X}\left(K_{j}, K\right) \rightarrow 0
$$

for $t$ in $\mathbb{R}^{+}$.

\subsection{Properties of $\Phi^{(n)}(t, x)$}

We prove further that the maps $\Phi^{(n)}(t, \cdot)$ are upper semi-continuous and have compact values.

Proposition 5 Suppose that $\Phi$ satisfies Assumptions A1-A2. Then $\Phi^{(n)}(t, x)$ is compact for all $t \geq 0$ and all $x \in X$.

Proof. Let $n=1$ and $t \in[0,1]$. If $t \in\left[0,2^{-1}\right]$, then $\Phi^{(1)}(t, x)=\Phi(t, x)$ and the result follows by the assumptions. On the other hand if $t \in\left[2^{-1}, 1\right]$, then $\Phi^{(1)}(t, x)=\Phi\left(t-2^{-1}, \Phi\left(2^{-1}, x\right)\right)=\Phi\left(t-2^{-1}, K\right)$ with $K=\Phi\left(2^{-1}, x\right)$ compact. Then the result follows by the assumptions.

Now let $n \geq 2$. If $t \in\left[0,2^{-n}\right]$, then $\Phi^{(n)}(t, x)=\Phi(t, x)$ and the result follows by the assumptions. On the other hand if $t \in\left[j 2^{-n},(j+1) 2^{-n}\right]$, then

$$
\Phi^{(n)}(t, x)=\Phi\left(t-j 2^{-n}, \Phi^{(n-1)}\left(j 2^{-n}, x\right)\right)=\Phi\left(t-j 2^{-n}, K_{j}^{(n)}\right)
$$

with $K_{j}^{(n)}=\Phi^{(n-1)}\left(j 2^{-n}, x\right)=\Phi\left(2^{-n}, K_{j-1}^{(n)}\right)$, writing $K_{j-1}^{(n)}=\Phi^{(n-2)}\left((j-1) 2^{-n}, x\right)$, and $K_{0}^{(n)}=\{x\}$.

Inductively, by the assumptions of $\Phi$, we obtain that the sets $K_{j}^{(n)}$ are compact, and then that $\Phi^{(n)}(t, x)$ is compact.

Proposition 6 Suppose that $\Phi$ satisfies Assumptions A1-A2. Then the multivalued mapping $x \rightarrow$ $\Phi^{(n)}(t, x)$ is upper semi-continuous in $x$ for each $t$ in $\mathbb{R}^{+}$and each $n \in \mathbb{N}$.

Proof. Let $n \geq 1$ be fixed and let $x_{k} \rightarrow x^{*}$. Define $K_{0, k}^{(n)}:=\left\{x_{k}\right\}$ and $K_{0}^{(n)}:=\left\{x^{*}\right\}$. Then $\operatorname{dist}_{X}\left(K_{0, k}^{(n)}, K_{0}^{(n)}\right)$ $\rightarrow 0$ as $k \rightarrow \infty$. If $t \in\left[0,2^{-n}\right]$, then

$$
\Phi^{(n)}\left(t, x_{k}\right)=\Phi\left(t, x_{k}\right)=\Phi\left(t, K_{0, k}^{(n)}\right) \rightarrow \Phi\left(t, K_{0}^{(n)}\right)=\Phi\left(t, x^{*}\right)=\Phi^{(n)}\left(t, x^{*}\right)
$$

as $k \rightarrow \infty$ in the upper semi-continuous sense (4). In particular, for $t=2^{-n}$ and $k \rightarrow \infty$,

$$
K_{1, k}^{(n)}:=\Phi\left(2^{-n}, K_{0, k}^{(n)}\right) \rightarrow \Phi\left(2^{-n}, K_{0}^{(n)}\right)=: K_{1}^{(n)} .
$$

The proof proceeds inductively. Define

$$
K_{j, k}^{(n)}:=\Phi\left(2^{-n}, K_{j-1, k}^{(n)}\right), \quad K_{j}^{(n)}:=\Phi\left(2^{-n}, K_{j-1}^{(n)}\right) .
$$

Then for $t \in\left[j 2^{-n},(j+1) 2^{-n}\right]$ and $k \rightarrow \infty$,

$$
\begin{aligned}
\Phi^{(n)}\left(t, x_{k}\right) & =\Phi\left(t-j 2^{-n}, \Phi^{(n-1)}\left(j 2^{-n}, x_{k}\right)\right) \\
& =\Phi\left(t-j 2^{-n}, K_{j, k}^{(n)}\right) \\
& \rightarrow \Phi\left(t-j 2^{-n}, K_{j}^{(n)}\right)=\Phi^{(n)}\left(t, x^{*}\right)
\end{aligned}
$$


and, in particular,

$$
K_{j+1, k}^{(n)}:=\Phi\left(\left(2^{-n}, K_{j, k}^{(n)}\right) \rightarrow \Phi\left(2^{-n}, K_{j}^{(n)}\right)=: K_{j+1}^{(n)} .\right.
$$

In summary, $\Phi^{(n)}$ is a non-strict multivalued dynamical system satisfying Assumptions A1-A2, which is strict at the $n$-dyadic points.

\subsection{Extensions of the maps $\Phi^{(n)}$ to the whole of $\mathbb{R}^{+}$}

The mappings $\Phi^{(n)}$ will be extended to the whole of $\mathbb{R}^{+}$with the following construction. Let $\Phi_{T}^{(n)}$ denote the counterpart of $\Phi^{(n)}$ defined on the interval $[0, T]$ with the dyadics $\left\{j 2^{-n} T, j=0,1, \ldots, 2^{n}\right\}$ instead of on the interval $[0,1]$. In this section we will use intervals of the form $\left[0,2^{m}\right]$. For notational convenience we write $\Phi_{m}^{(n)}$ instead of $\Phi_{2^{m}}^{(n)}$. Note that $\Phi_{m}^{(m+n)}(t, x)=\Phi_{m-1}^{(m+n-1)}(t, x)$ if $0 \leq t \leq 2^{m-1}$ for each $m \in \mathbb{N}$.

For $n=0,1$ we define the multivalued mappings $\bar{\Phi}^{(0)}(t, x), \bar{\Phi}^{(1)}(t, x)$ for all $x \in X$ and $t \in \mathbb{R}^{+}$by

$$
\begin{array}{cc}
\bar{\Phi}^{(0)}(t, x)=\Phi_{m}^{(m)}(t, x) & \text { if } 0 \leq t \leq 2^{m}, \quad m=0,1,2 \cdots \\
\bar{\Phi}^{(1)}(t, x)=\Phi_{m}^{(1+m)}(t, x) & \text { if } 0 \leq t \leq 2^{m}, \quad m=0,1,2 \cdots
\end{array}
$$

Similarly, for $n=2$ we define the multivalued mapping $\bar{\Phi}^{(2)}(t, x)$ for all $x \in X$ and $t \in \mathbb{R}^{+}$by

$$
\bar{\Phi}^{(2)}(t, x)=\Phi_{m}^{(2+m)}(t, x) \quad \text { if } 0 \leq t \leq 2^{m}, \quad m=0,1,2 \cdots,
$$

and for a general $n \geq 2$ we define the multivalued mapping $\bar{\Phi}^{(n)}(t, x)$ for all $x \in X$ and $t \in \mathbb{R}^{+}$by

$$
\bar{\Phi}^{(n)}(t, x)=\Phi_{m}^{(n+m)}(t, x) \quad \text { if } 0 \leq t \leq 2^{m}, \quad m=0,1,2 \cdots
$$

Since the maps $\Phi_{m}^{(n)}$ are strict semiflows at the dyadic points, it is clear that

$$
\bar{\Phi}^{(n)}(t+s, x)=\bar{\Phi}^{(n)}\left(t, \bar{\Phi}^{(n)}(s, x)\right),
$$

for all $s, t \in \widehat{\mathfrak{D}}_{n}:=\left\{j 2^{-n}: j=0,1,2, \ldots\right\}$.

Finally, we define $\Phi^{(\infty)}(t, x)$ by

$$
\Phi^{(\infty)}(t, x):=\bigcup_{n \in \mathbb{N}} \bar{\Phi}^{(n)}(t, x)
$$

This multivalued semiflow $\Phi^{(\infty)}$ is strict at $\widehat{\mathfrak{D}}_{n}$, i.e., all $2^{n}$-dyadics in $\mathbb{R}^{+}$. Indeed, if $s, t \in \widehat{\mathfrak{D}}_{m} \subset$ $\widehat{\mathfrak{D}}_{m+k}, k=1,2, \ldots$, then

$$
\begin{aligned}
\Phi^{(\infty)}(t+s, x) & =\bigcup_{n} \bar{\Phi}^{(n)}(t+s, x) \\
& =\bigcup_{n \geq m} \bar{\Phi}^{(n)}(t+s, x) \\
& =\bigcup_{n \geq m} \bar{\Phi}^{(n)}\left(t, \bar{\Phi}^{(n)}(s, x)\right) \\
& =\bigcup_{n \geq m} \bar{\Phi}^{(n)}\left(t, \bigcup_{k \geq n} \bar{\Phi}^{(k)}(s, x)\right)=\Phi^{(\infty)}\left(t, \Phi^{(\infty)}(s, x)\right) .
\end{aligned}
$$

Here we have used the fact that

$$
\bar{\Phi}^{(n)}\left(t, \bar{\Phi}^{(k)}(s, x)\right) \subset \bar{\Phi}^{(k)}\left(t, \bar{\Phi}^{(k)}(s, x)\right)
$$

if $k \geq n$. 


\subsection{Global attractor for $\bar{\Phi}^{(n)}$}

Suppose now that $\Phi(t, x)$ has a bounded, positively semi-invariant absorbing set $B$, i.e., $\Phi(t, B) \subset B$ for all $t \geq 0$ and for each bounded subset $D$ of $X$ there exists $T_{D} \geq 0$ such that $\Phi(t, D) \subset B$ for all $t \geq T_{D}$, and that $\Phi(t, \cdot)$ is a compact operator if $t>0$ (rather than being asymptotically compact in the sense of Theorem 3$)$.

If Assumptions A1-A2 hold, then $\Phi$ has a global compact attractor $\mathcal{A}^{(0)}$ by Theorem 3 and, moreover, it follows from $\Phi(t, B) \subset B$ that $\mathcal{A}^{(0)}:=\bigcap_{t \geq 0} \Phi(t, B) \subset B$.

Consider a fixed but arbitrary $n \in \mathbb{N}$. Assume that the set $B$ is also absorbing for $\bar{\Phi}^{(n)}$. Then, by its construction, $\bar{\Phi}^{(n)}(t, B) \subset B$ for all $t \geq 0, \bar{\Phi}^{(n)}(t, \cdot)$ is compact if $t>0$ and it satisfies Assumptions A1-A2 (by Propositions 5, 6), so

$$
\mathcal{A}^{(n)}:=\bigcap_{t \geq 0} \bar{\Phi}^{(n)}(t, B)
$$

is a global compact attractor of $\bar{\Phi}^{(n)}$.

In general, $\mathcal{A}^{(n)} \subseteq \bar{\Phi}^{(n)}\left(t, \mathcal{A}^{(n)}\right)$ for $t \in \mathbb{R}^{+}$, but the inclusion is strict for the $n$-dyadics, i.e.,

$$
\mathcal{A}^{(n)}=\bar{\Phi}^{(n)}\left(t_{j}, \mathcal{A}^{(n)}\right)
$$

for the $t_{j} \in \widehat{\mathfrak{D}}_{n}$. Indeed, this follows from

$$
\bar{\Phi}^{(n)}\left(t_{j}, \mathcal{A}^{(n)}\right) \subset \bar{\Phi}^{(n)}\left(t_{j}, \bar{\Phi}^{(n)}\left(\tau_{k}, \mathcal{A}^{(n)}\right)\right)=\bar{\Phi}^{(n)}\left(t_{j}+\tau_{k}, \mathcal{A}^{(n)}\right) \rightarrow \mathcal{A}^{(n)} \text { as } \tau_{k} \rightarrow \infty,
$$

where $\tau_{k} \in \widehat{\mathfrak{D}}_{n}$.

In particular,

$$
\mathcal{A}^{(n)} \subseteq \mathcal{A}^{(n+1)} \subseteq B
$$

for $n=0,1,2, \ldots$, which follows from

$$
\mathcal{A}^{(n)}=\bigcap_{t \geq 0} \bar{\Phi}^{(n)}(t, B) \subseteq \bigcap_{t \geq 0} \bar{\Phi}^{(n+1)}(t, B)=\mathcal{A}^{(n+1)}
$$

since $\bar{\Phi}^{(n)}(t, x) \subseteq \bar{\Phi}^{(n+1)}(t, x)$ for all $x \in X, t \geq 0$ and $n=0,1,2, \ldots$

The set defined by

$$
\mathcal{A}^{(\infty)}:=\bigcup_{n=1}^{\infty} \mathcal{A}^{(n)} \subseteq B
$$

corresponds to the set of all possible limit points of the maps $\bar{\Phi}^{(n)}$ as $\Phi$ undergoes a countable number of "switchings" with a fixed dyadic time step.

Proposition 7 Let Assumptions A1-A2 hold. Assume that $\Phi(t, \cdot)$ is a compact operator if $t>0$ and that $B$ is a bounded positively invariant absorbing set for all $\Phi^{(n)}, n=0,1, \cdots$. Then $\mathcal{A}^{(\infty)}$ is strictly invariant for $\Phi^{(\infty)}$ on the dyadics.

Proof. We want to prove that

$$
\mathcal{A}^{(\infty)}=\Phi^{(\infty)}\left(t, \mathcal{A}^{(\infty)}\right)
$$

for all $t \in \widehat{\mathfrak{D}}=\cup_{n} \widehat{\mathfrak{D}}_{n}$.

From a general identity we have

$$
\Phi^{(\infty)}\left(t, \mathcal{A}^{(\infty)}\right):=\Phi^{(\infty)}\left(t, \cup_{n} \mathcal{A}^{(n)}\right) \equiv \bigcup_{n} \Phi^{(\infty)}\left(t, \mathcal{A}^{(n)}\right) .
$$


Since the $\Phi^{(n)}(t, x)$ and $\mathcal{A}^{(n)}$ are increasing in $n$ we have

$$
\begin{aligned}
\mathcal{A}^{(n)} \subset \bar{\Phi}^{(n)}\left(t, \mathcal{A}^{(n)}\right) & \subset \bigcup_{k \geq n} \bar{\Phi}^{(k)}\left(t, \mathcal{A}^{(k)}\right) \\
& \subset \bigcup_{k \geq n} \Phi^{(\infty)}\left(t, \mathcal{A}^{(k)}\right) \\
& \subset \Phi^{(\infty)}\left(t, \mathcal{A}^{(\infty)}\right) \quad \text { for all } t \geq 0,
\end{aligned}
$$

from which it follows that

$$
\bigcup_{n=1}^{\infty} \mathcal{A}^{(n)}=: \mathcal{A}^{(\infty)} \subseteq \Phi^{(\infty)}\left(t, \mathcal{A}^{(\infty)}\right) \quad \text { for all } t \geq 0 .
$$

Now let $t \in \widehat{\mathfrak{D}}_{n}$ for some $n \in \mathbb{N}$ and let $x \in \Phi^{(\infty)}\left(t, \mathcal{A}^{(\infty)}\right)$. Then there exists an $n^{*} \geq n$ such that $x \in \Phi^{\left(n^{*}\right)}\left(t, \mathcal{A}^{\left(n^{*}\right)}\right) \subset \Phi^{(\infty)}\left(t, \mathcal{A}^{(\infty)}\right)$. Moreover, $t \in \widehat{\mathfrak{D}}_{n} \subset \widehat{\mathfrak{D}}_{n^{*}}$. Hence there exists an $x^{*} \in \mathcal{A}^{\left(n^{*}\right)}$ such that $x \in \Phi^{\left(n^{*}\right)}\left(t, x^{*}\right) \subset \Phi^{\left(n^{*}\right)}\left(t, \mathcal{A}^{\left(n^{*}\right)}\right)$. Since $\mathcal{A}^{\left(n^{*}\right)}$ is $\Phi^{\left(n^{*}\right)}$-invariant for all $t \in \widehat{\mathfrak{D}}_{n^{*}}$, we have $x \in$ $\Phi^{\left(n^{*}\right)}\left(t, \mathcal{A}^{\left(n^{*}\right)}\right)=\mathcal{A}^{\left(n^{*}\right)}$. Hence $x \in \mathcal{A}^{(\infty)}$. But $x \in \Phi^{(\infty)}\left(t, \mathcal{A}^{(\infty)}\right)$ was chosen arbitrarily, so we have $\Phi^{(\infty)}\left(t, \mathcal{A}^{(\infty)}\right) \subset \mathcal{A}^{(\infty)}$.

Remark 8 The assumption that $B$ is a bounded positively semi-invariant absorbing set for all $\bar{\Phi}^{(n)}$ is quite natural in applications. We consider the usual case where $X$ is a Banach space with norm $\|\cdot\|$ and $\Phi$ satisfies the estimate

$$
\|y\|^{2} \leq\|x\|^{2} e^{-\alpha t}+R_{0}^{2}\left(1-e^{-\alpha t}\right), \text { for all } y \in \Phi(t, x) \text { and } t \geq 0 .
$$

Then the set $B=\left\{x \in X:\|x\|^{2} \leq R_{0}^{2}+1\right\}$ is a bounded positively semi-invariant absorbing set for $\Phi$. We shall prove that

$$
\|y\|^{2} \leq\|x\|^{2} e^{-\alpha t}+R_{0}^{2}\left(1-e^{-\alpha t}\right), \text { for all } y \in \bar{\Phi}^{(n)}(t, x) \text { and } t \geq 0 .
$$

We proceed inductively. Assume that (6) holds for $\bar{\Phi}^{(n-1)}$. Since any $z \in \bar{\Phi}^{(n)}(t, x)$ with $j 2^{-n}<t \leq$ $(j+1) 2^{-n}$ satisfies

$$
z \in \Phi\left(t-j 2^{-n}, \Phi^{(n-1)}\left(j 2^{-n}, x\right)\right)
$$

we have

$$
\begin{aligned}
\|z\|^{2} & \leq \sup _{y \in \Phi^{(n-1)}\left(j 2^{-n}, x\right)}\|y\|^{2} e^{-\alpha\left(t-j 2^{-n}\right)}+R_{0}^{2}\left(1-e^{-\alpha\left(t-j 2^{-n}\right)}\right) \\
& \leq\left(\|x\|^{2} e^{-\alpha\left(j 2^{-n}\right)}+R_{0}^{2}\left(1-e^{-\alpha\left(j 2^{-n}\right)}\right)\right) e^{-\alpha\left(t-j 2^{-n}\right)}+R_{0}^{2}\left(1-e^{-\alpha\left(t-j 2^{-n}\right)}\right) \\
& \leq\|x\|^{2} e^{-\alpha t}+R_{0}^{2}\left(1-e^{-\alpha t}\right) .
\end{aligned}
$$

Hence, B is a bounded, positively semi-invariant absorbing set for all $\bar{\Phi}^{(n)}$.

\subsection{Independence of discretization interval}

The envelope attractor $\mathcal{A}^{(\infty)}$ of $\Phi$ is independent of the basic time interval used for the dyadic partition.

Suppose we use $[0, T]$ instead of $[0,1]$, where $T>1$, and let $\Phi_{T}^{(n)}$ be the counterpart of the mapping $\Phi^{(n)}$ constructed over $[0, T]$ instead of $[0,1]$. 
Since $2^{-1}<2^{-1} T$ we have $\bar{\Phi}_{T}^{(n)}(t, x)=\bar{\Phi}^{(n)}(t, x)=\Phi(t, x)$ for $0 \leq t \leq 2^{-n}$ and

$$
\bar{\Phi}_{T}^{(n)}(t, x)=\Phi(t, x) \subset \bar{\Phi}^{(n)}(t, x) \quad \text { for } 2^{-n} \leq t \leq 2^{-n} T,
$$

from which it follows that $\bar{\Phi}_{T}^{(n)}(t, x) \subset \bar{\Phi}^{(n)}(t, x)$ for all $t \geq 0$.

Now let $k$ be the first integer such that $2^{-n-k} T \leq 2^{-n}$. Reversing the roles of $T$ and 1 in the previous argument, we obtain

$$
\bar{\Phi}^{(n)}(t, x) \subset \bar{\Phi}_{T}^{(n+k)}(t, x) \subset \bar{\Phi}^{(n+k)}(t, x)
$$

for all $t \geq 0$. This gives

$$
\bar{\Phi}_{T}^{(n)}(t, x) \subset \bar{\Phi}^{(n)}(t, x) \subset \bar{\Phi}_{T}^{(n+k)}(t, x) \subset \bar{\Phi}^{(n+k)}(t, x) \quad \text { for all } t \geq 0, x \in X .
$$

If $T<1$, then a similar argument gives

$$
\bar{\Phi}^{(n)}(t, x) \subset \bar{\Phi}_{T}^{(n)}(t, x) \subset \bar{\Phi}^{(n+k)}(t, x) \subset \bar{\Phi}_{T}^{(n+k)}(t, x) \text { for all } t \geq 0, x \in X .
$$

Since the attractors satisfy a similar subset relationship to the multivalued mappings, we have

$$
\mathcal{A}_{T}^{(n)} \subset \mathcal{A}^{(n)} \subset \mathcal{A}_{T}^{(n+k)} \subset \mathcal{A}^{(n+k)}
$$

in the first case and

$$
\mathcal{A}^{(n)} \subset \mathcal{A}_{T}^{(n)} \subset \mathcal{A}^{(n+k)} \subset \mathcal{A}_{T}^{(n+k)}
$$

in the second case. These give

$$
\mathcal{A}^{(\infty)}=\bigcup_{n=1}^{\infty} \mathcal{A}^{(n)}=\bigcup_{n=1}^{\infty} \mathcal{A}_{T}^{(n)}=\mathcal{A}_{T}^{(\infty)}
$$

\subsection{An envelope system: a first attempt}

The (on dyadics) invariant set $\mathcal{A}^{(\infty)}=\cup_{n=1}^{\infty} \mathcal{A}^{(n)}$ may not be an attractor of the envelope mapping $\Phi^{(\infty)}$.

Hence consider $\widehat{X}:=\prod_{i=0}^{\infty} X$ (countable cartesian product of $X$ ) with the product topology defined by the metric

$$
\rho_{\widehat{X}}(x, y)=\sum_{i=0}^{\infty} 2^{-i} \frac{\rho\left(x_{i}, y_{i}\right)}{1+\rho\left(x_{i}, y_{i}\right)} .
$$

This means that $x^{n} \rightarrow x$ if $x_{i}^{n} \rightarrow x_{i}$ in $X$ for every $i \in \mathbb{N}$.

Further, we define the multivalued map $\widehat{\Phi}^{(\infty)}(\hat{x})$ on $\widehat{X}$ component-wise as the cartesian product of the time-one maps $\Phi^{(n)}(1, x)$ by

$$
\widehat{\Phi}^{(\infty)}(\hat{x}):=\left(\bar{\Phi}^{(0)}\left(1, x_{0}\right), \bar{\Phi}^{(1)}\left(1, x_{1}\right), \ldots, \bar{\Phi}^{(n)}\left(1, x_{n}\right), \ldots\right),
$$

where $\hat{x}:=\left(x_{0}, x_{1}, \ldots, x_{n}, \ldots\right)$.

Clearly, $\widehat{\mathcal{A}}:=\mathcal{A}^{(0)} \times \mathcal{A}^{(1)} \times \cdots \times \mathcal{A}^{(n)} \times \cdots$ is a strictly invariant compact global attractor for the time-one map $\widehat{\Phi}^{(\infty)}$.

The set $\widehat{\mathcal{A}}$ thus serves as an attractor for the dynamics with a countable number of "switches" with a fixed time step in any finite interval at the corresponding dyadics, but the construction is somewhat artificial. 


\subsection{An envelope system in $X$}

We consider the multivalued semiflows $\Phi^{(\infty)}, \bar{\Phi}^{(\infty)}: X \rightarrow \mathcal{P}(X)$ given by

$$
\Phi^{(\infty)}(t, x)=\bigcup_{n} \bar{\Phi}^{(n)}(t, x)
$$

and

$$
\bar{\Phi}^{(\infty)}(t, x)=\left\{y: \exists x_{n} \rightarrow x, t_{n} \rightarrow t, y_{n} \in \Phi^{\infty}\left(t_{n}, x_{n}\right) \text { such that } y_{n} \rightarrow y\right\} .
$$

We know, moreover, that $\Phi^{(\infty)}$ is strict on $\widehat{\mathfrak{D}}:=\cup_{n} \widehat{\mathfrak{D}}_{n}$.

Lemma 9 The map $(t, x) \mapsto \bar{\Phi}^{(\infty)}(t, x)$ has a closed graph.

Proof. Let $z_{n} \in \bar{\Phi}^{(\infty)}\left(t_{n}, x_{n}\right)$ be such that $z_{n} \rightarrow z, t_{n} \rightarrow t$ and $x_{n} \rightarrow x$. Then there exist $\tau_{n}, u_{n}$ and $\xi_{n}$ $\in \Phi^{\infty}\left(\tau_{n}, u_{n}\right)$ such that

$$
\rho\left(\xi_{n}, z_{n}\right)<\frac{1}{n}, \quad \rho\left(\tau_{n}, t_{n}\right)<\frac{1}{n}, \quad \rho\left(u_{n}, x_{n}\right)<\frac{1}{n} .
$$

Hence, $\xi_{n} \rightarrow z, u_{n} \rightarrow x$ and $\tau_{n} \rightarrow t$, from which it follows that $z \in \bar{\Phi}^{(\infty)}(t, x)$, i.e., the graph is closed.

If we assume that $\bar{\Phi}^{(\infty)}$ has a compact attracting set $K$, then by Lemma 9 , Theorem 3 and Remark 4 it possesses the global compact attractor

$$
\widetilde{\mathcal{A}}=\bigcap_{t \geq 0} \overline{\bigcup_{\tau \geq t} \bar{\Phi}^{(\infty)}(\tau, K)}
$$

Also, for $\Phi^{(\infty)}$ the omega-limit set of the attracting set

$$
\mathcal{A}=\bigcap_{t \geq 0} \overline{\bigcup_{\tau \geq t} \Phi^{(\infty)}(\tau, K)}
$$

is another compact attracting set.

We will prove that $\mathcal{A}=\widetilde{\mathcal{A}}$. Since $\mathcal{A} \subset \widetilde{\mathcal{A}}$ is obvious, we need to check that $\widetilde{\mathcal{A}} \subset \mathcal{A}$. Let $y \in \widetilde{\mathcal{A}}$. Then $y=\lim y_{n}$, where $y_{n} \in \bar{\Phi}^{(\infty)}\left(t_{n}, x_{n}\right)$ and $t_{n} \rightarrow \infty$. Hence, there exist $z_{n} \in \Phi^{(\infty)}\left(t_{n}, u_{n}\right)$ with $\rho\left(y_{n}, z_{n}\right)$ $<\frac{1}{n}$ and $\rho\left(u_{n}, x_{n}\right)<\frac{1}{n}$. Then, it is clear that

$$
\rho\left(y, z_{n}\right) \leq \rho\left(y, y_{n}\right)+\rho\left(y_{n}, z_{n}\right) \rightarrow 0,
$$

so that $y \in \mathcal{A}$.

We note that $\mathcal{A} \subset \bar{\Phi}^{(\infty)}(t, \mathcal{A})$, since the global attractor is, by definition, negatively semi-invariant for $\bar{\Phi}^{(\infty)}$.

\subsection{Weak positive semi-invariance of the attractor}

Let $\mathcal{R} \subset C\left(\mathbb{R}^{+} ; X\right)$ be some set of functions such that the following conditions hold:

(K1) For any $x \in X$ there exists $\varphi \in \mathcal{R}$ such that $\varphi(0)=x$.

$(K 2) \varphi_{\tau}(\cdot)=\varphi(\cdot+\tau) \in \mathcal{R}$ for any $\tau \geq 0, \varphi(\cdot) \in \mathcal{R}$ (translation property). 
Assume that the multivalued semiflow $\Phi$ is generated by $\mathcal{R}$, that is,

$$
y \in \Phi(t, x) \text { if } \exists \varphi \in \mathcal{R} \text { such that } y=\varphi(t), \varphi(0)=x
$$

Any map $\varphi \in \mathcal{R}$ is called a trajectory of the multivalued semiflow $\Phi$. It is easy to see that any $\varphi \in \mathcal{R}$ satisfies

$$
\varphi(t+s) \in \Phi(t, \varphi(s)), \forall t, s \in \mathbb{R}^{+}
$$

Any map $\varphi \in C\left(\mathbb{R}^{+} ; X\right)$ such that $(8)$ holds is called a $\Phi$-trajectory. Obviously, every trajectory is a $\Phi$-trajectory, but the converse is not necessarily true in general, although this is the case under extra assumptions (see [8]).

A map $\psi: \mathbb{R}^{+} \rightarrow X$ is called a continuous pseudo-trajectory of the multivalued semiflow $\Phi$ if it is a concatenation of a countable number of trajectories, that is, if there exist a sequence of trajectories $\varphi_{m} \in \mathcal{R}$ and a sequence of points $0=t_{0}<t_{1}<t_{2}<\cdots<t_{m}<\cdots$ such that

$$
\psi(t)=\varphi_{m}\left(t-t_{m-1}\right) \quad \text { for } t_{m-1} \leq t \leq t_{m}, \quad m \in \mathbb{N}
$$

(This requires that $\varphi_{m+1}(0)=\varphi_{m}\left(t_{m}-t_{m-1}\right)$ for $\left.m \in \mathbb{N}\right)$. We note that when the concatenation of trajectories is again a trajectory (see condition (K3) below), a continuous pseudo-trajectory is also a trajectory.

We assume the following condition:

Assumption A3: If $\left\{\psi_{n}(\cdot)\right\}$ is a sequence of continuous pseudo-trajectories with $\psi_{n}(0)=x_{n}$ and $x_{n} \rightarrow$ $x$, then there exists a subsequence such that

$$
\psi_{n}\left(t_{n}\right) \rightarrow \phi(t) \text { if } t_{n} \rightarrow t
$$

where $\phi: \mathbb{R}^{+} \rightarrow X$ is a continuous function such that $\phi(0)=x$.

Note that, in general, $\phi$ here need not be a pseudo-trajectory.

Lemma 10 Let Assumption A3 and (7) hold. Then $\bar{\Phi}^{(\infty)}(t, K)$ is compact for any compact set $K$.

Proof. Let $y_{n} \in \bar{\Phi}^{(\infty)}\left(t, x_{n}\right)$ with $x_{n} \in K$. Then there exist $\tau_{n}, u_{n}$ and $\xi_{n} \in \Phi^{(\infty)}\left(\tau_{n}, u_{n}\right)$ such that

$$
\rho\left(\xi_{n}, y_{n}\right)<\frac{1}{n}, \quad \rho\left(\tau_{n}, t\right)<\frac{1}{n}, \quad \rho\left(u_{n}, x_{n}\right)<\frac{1}{n} .
$$

Hence, $\xi_{n} \in \bar{\Phi}^{\left(k_{n}\right)}\left(\tau_{n}, u_{n}\right)$ for some $k_{n} \in \mathbb{N}$. In view of the definition of $\bar{\Phi}^{\left(k_{n}\right)}$ and (7), we have

$$
\xi_{n}=\psi_{n}\left(\tau_{n}\right)
$$

where $\psi_{n}(\cdot)$ is a continuous pseudo-trajectory such that $\psi_{n}(0)=u_{n}$. In view of Assumption A3 and Lemma 9, we also have, up to a subsequence,

$$
x_{n} \rightarrow x, \quad \psi_{n}\left(\tau_{n}\right) \rightarrow \phi(t) \in \bar{\Phi}^{(\infty)}(t, x) .
$$

Thus, up to a subsequence, $y_{n} \rightarrow \phi(t) \in \bar{\Phi}^{(\infty)}(t, x) \subset \bar{\Phi}^{(\infty)}(t, K)$.

Lemma 11 Assume that $\Phi$ satisfies Assumption A3 and that (7) holds. Then the map $(t, x) \mapsto \bar{\Phi}^{(\infty)}(t, x)$ is upper semi-continuous. 
Proof. If we assume that this is not true, then there exist a neighbourhood $O$ of $\bar{\Phi}^{(\infty)}(t, x)$ and sequences $t_{n} \rightarrow t, x_{n} \rightarrow x$ and $y_{n} \in \bar{\Phi}^{(\infty)}\left(t_{n}, x_{n}\right)$ such that $y_{n} \notin O$. Hence, there are $\tau_{n}, u_{n}$ and $\xi_{n} \in \Phi^{\left(k_{n}\right)}\left(\tau_{n}, u_{n}\right)$, where $k_{n} \in \mathbb{N}$, such that

$$
\rho\left(\xi_{n}, y_{n}\right)<\frac{1}{n}, \quad \rho\left(\tau_{n}, t\right)<\frac{1}{n}, \quad \rho\left(u_{n}, x_{n}\right)<\frac{1}{n} .
$$

In view of (7), we have

$$
\xi_{n}=\psi_{n}\left(\tau_{n}\right),
$$

where $\psi_{n}(\cdot)$ is a continuous pseudo-trajectory such that $\psi_{n}(0)=u_{n}$. Assumption A3 and Lemma 9 imply that for some subsequence,

$$
x_{n} \rightarrow x, \quad \psi_{n}\left(\tau_{n}\right) \rightarrow \phi(t) \in \bar{\Phi}^{(\infty)}(t, x) .
$$

Thus, $y_{n} \rightarrow \phi(t) \in \bar{\Phi}^{(\infty)}(t, x)$, which is a contradiction.

Corollary 12 Assume that $\Phi$ satisfies Assumption A3 and that (7) holds. Then the map $(t, x) \mapsto$ $\Phi^{(\infty)}(t, x)$ is upper semi-continuous.

Proof. Take an arbitrary $(t, x)$ and a neighborhood $O$ of $\Phi^{(\infty)}(t, x)$, which is also a neighborhood of $\bar{\Phi}^{(\infty)}(t, x)$. Then, as $(t, x) \mapsto \bar{\Phi}^{(\infty)}(t, x)$ is upper semi-continuous, there exists a $\delta>0$ such that if $\rho(y, x)$ $<\delta$ and $\rho(\tau, t)<\delta$, then

$$
\Phi^{(\infty)}(\tau, y) \subset \bar{\Phi}^{(\infty)}(t, x) \subset O
$$

We have seen in Section 3.6 that if $\bar{\Phi}^{(\infty)}$ has a compact attracting set $B$, then $\bar{\Phi}^{(\infty)}$ possesses a global compact attractor $\mathcal{A}$, which is equal to the omega-limit set of $B$ for the map $\Phi^{(\infty)}$ :

$$
\mathcal{A}=\bigcap_{t \geq 0} \overline{\bigcup_{\tau \geq t} \Phi^{(\infty)}(\tau, B)}
$$

We say that the attractor $\mathcal{A}$ is weakly positively semi-invariant if for any $y \in A$ there exists a $\bar{\Phi}^{(\infty)}$ trajectory $\phi$ starting at $y$ such that $\phi(t) \in A$ for all $t \geq 0$, i.e., for each point in $\mathcal{A}$ there is at least one $\bar{\Phi}^{(\infty)}$-trajectory that stays in $\mathcal{A}$.

We prove that the attractor $\mathcal{A}$ is weakly positively semi-invariant for $\bar{\Phi}^{(\infty)}$. See [13] for strict multivalued semiflows.

Theorem 13 Assume that $\bar{\Phi}^{(\infty)}$ has a compact attracting set B. Let (7) and Assumption A3 hold. Then the multivalued semiflow $\Phi^{(\infty)}$ has a global compact attractor $\mathcal{A}$, which is weakly positively semi-invariant, that is, for any $y \in \mathcal{A}$ there exists a continuous function $\phi$ such that $\phi(0)=y$ and $\phi(t) \in \mathcal{A}$ for all $t \geq$ 0 with

$$
\phi(t+s) \in \bar{\Phi}^{(\infty)}(t, \phi(s)) \text { for all } t, s \geq 0
$$

Proof. Let $y \in \mathcal{A}$ be arbitrary. We prove the existence of a $\bar{\Phi}^{(\infty)}$-trajectory $\phi$ of the multivalued semiflow $\bar{\Phi}^{(\infty)}$ such that $\phi(t) \in \mathcal{A}$ for all $t \geq 0$ and $\phi(0)=y$.

Since $y \in \mathcal{A}$, there exists a sequence $\xi_{n} \in \Phi^{(\infty)}\left(t_{n}, B\right)$, where $t_{n} \rightarrow \infty$, such that $\xi_{n} \rightarrow y$. Thus $\xi_{n} \in \bar{\Phi}^{\left(k_{n}\right)}\left(t_{n}, B\right)$ for some $k_{n} \in \mathbb{N}$. By the definition of $\bar{\Phi}^{\left(k_{n}\right)}$ there exists a $k_{n}$-dyadic point $\tau_{n} \in \widehat{\mathfrak{D}}_{k_{n}}$ with $t_{n}-2^{-k_{n}} \leq \tau_{n}<t_{n}$ such that $\xi_{n} \in \Phi\left(t_{n}-\tau_{n}, z_{n}\right)$, where $z_{n} \in \bar{\Phi}^{\left(k_{n}-1\right)}\left(\tau_{n}, B\right)$. Then there exists a trajectory $\eta_{n}$ of $\Phi$ such that $\eta_{n}(0)=z_{n}$ and $\eta_{n}\left(t_{n}-\tau_{n}\right)=\xi_{n}$. We choose a dyadic point $\bar{t}_{n} \in \widehat{\mathfrak{D}}=$ 
$\cup_{m} \widehat{\mathfrak{D}}_{m}$ with $\tau_{n}<\bar{t}_{n}<t_{n}$ such that $y_{n}=\eta_{n}\left(\bar{t}_{n}-\tau_{n}\right)$ satisfies $\rho\left(y_{n}, \xi_{n}\right)<\frac{1}{n}$. Hence, $y_{n} \rightarrow y$ and $y_{n} \in$ $\bar{\Phi}^{\left(k_{n}\right)}\left(\bar{t}_{n}, B\right)$ with $\bar{t}_{n} \rightarrow \infty$ and $\bar{t}_{n} \in \widehat{\mathfrak{D}}$.

We will construct a continuous pseudo-trajectory starting at $y_{n}$ in the following way: we take trajectories $\varphi_{j}^{n}$ of $\Phi$ such that

$$
\varphi_{1}^{n}(0)=y_{n}, \quad \varphi_{2}^{n}(0)=\varphi_{1}^{n}\left(2^{-k_{n}}\right), \cdots, \varphi_{j}^{n}(0)=\varphi_{j-1}^{n}\left(2^{-k_{n}}\right), \cdots,
$$

and define

$$
\psi_{n}(t)=\varphi_{j}^{n}\left(t-(j-1) 2^{-k_{n}}\right) \quad \text { if } \quad(j-1) 2^{-k_{n}} \leq t \leq j 2^{-k_{n}}, \quad j \in \mathbb{N} .
$$

From the definition of $\bar{\Phi}^{\left(k_{n}\right)}$ it is clear that $\psi_{n}(t) \in \bar{\Phi}^{\left(k_{n}\right)}\left(t, y_{n}\right)$ for all $t \geq 0$. By our assumptions there exists a continuous function $\phi$ with $\phi(0)=y$ and a subsequence of $\psi_{n}$ such that $\psi_{n}(t) \rightarrow \phi(t)$ for all $t \geq 0$. Since $\bar{\Phi}^{(\infty)}(t, \cdot)$ has closed graph by Lemma 9 , we have $\phi(t) \in \bar{\Phi}^{(\infty)}(t, y)$ for all $t \geq 0$.

Moreover, since $\Phi^{(\infty)}$ is strictly invariant at the dyadic points, it is clear that

$$
\psi_{n}(t) \in \bar{\Phi}^{\left(k_{n}\right)}\left(t, \bar{\Phi}^{\left(k_{n}\right)}\left(\bar{t}_{n}, B\right)\right) \subset \Phi^{(\infty)}\left(t, \Phi^{(\infty)}\left(\bar{t}_{n}, B\right)\right)=\Phi^{(\infty)}\left(t+\bar{t}_{n}, B\right)
$$

for any $t \in \widehat{\mathfrak{D}}$. It follows that $\phi(t) \in \mathcal{A}$ for all dyadic $t$ and hence, by the continuity of $\phi$, for all $t \geq 0$.

On the other hand, using the fact that the maps $\Phi^{(\infty)}$ are strictly invariant at any dyadic points, it is easy to check that

$$
\psi_{n}(t+s) \in \Phi^{(\infty)}\left(t, \psi_{n}(s)\right) \quad \text { for all } t, s \in \widehat{\mathfrak{D}} .
$$

Hence, since the graph of $(t, x) \mapsto \bar{\Phi}^{(\infty)}(t, x)$ is closed, we have

$$
\phi(t+s) \in \bar{\Phi}^{(\infty)}(t, \phi(s)) \quad \text { for all } t, s \in \widehat{\mathfrak{D}},
$$

as well as $\phi(t+s) \in \bar{\Phi}^{(\infty)}(t, \phi(s))$ for all $t, s \geq 0$.

\section{A strict envelope with an invariant attractor}

Let $\mathcal{G} \subset C\left(\mathbb{R}^{+} ; X\right)$ be some set of functions. We remember that the space $C\left(\mathbb{R}^{+} ; X\right)$ is metrizable and we can use the metric

$$
\rho_{C}(\psi, \varphi)=\sum_{i=0}^{\infty} 2^{-i} \frac{\sup _{t \in\left[0, T_{i}\right]} \rho(\varphi(t), \psi(t))}{1+\sup _{t \in\left[0, T_{i}\right]} \rho(\varphi(t), \psi(t))},
$$

for some arbitrary sequence $0<T_{0}<T_{1}<\ldots<T_{k}<\ldots, T_{k} \rightarrow \infty$.

It is useful to consider the following conditions:

$(K 1)$ For any $x \in X$ there exists $\varphi \in \mathcal{G}$ such that $\varphi(0)=x$.

$(K 2) \varphi_{\tau}(\cdot)=\varphi(\cdot+\tau) \in \mathcal{G}$ for any $\tau \geq 0, \varphi(\cdot) \in \mathcal{G}$ (translation property).

$(K 3)$ Let $\varphi_{1}, \varphi_{2} \in \mathcal{G}$ be such that $\varphi_{2}(0)=\varphi_{1}(s)$, where $s>0$. Then the function $\varphi(\cdot)$, defined by

$$
\varphi(t)=\left\{\begin{array}{l}
\varphi_{1}(t) \text { if } 0 \leq t \leq s \\
\varphi_{2}(t-s) \text { if } s \leq t
\end{array}\right.
$$

belongs to $\mathcal{G}$ (concatenation property). 
We define the multivalued map

$$
y \in \Phi(t, x) \text { if } \exists \varphi \in \mathcal{R} \text { such that } y=\varphi(t), \varphi(0)=x .
$$

It is straightforward to check that if $\mathcal{G}$ satisfies $(K 1)-(K 2)$, then $\Phi$ is a multivalued semiflow.

Any map $\varphi \in \mathcal{G}$ is called a trajectory of the multivalued semiflow $\Phi$. It is easy to see that any $\varphi \in \mathcal{G}$ satisfies

$$
\varphi(t+s) \in \Phi(t, \varphi(s)), \forall t, s \in \mathbb{R}^{+} .
$$

Let $\mathcal{R} \subset C\left(\mathbb{R}^{+} ; X\right)$ be some set of functions satisfying $(K 1)-(K 2)$. Let us consider also another set of functions $\mathcal{K} \subset C\left(\mathbb{R}^{+} ; X\right)$ such that $\mathcal{R} \subset \mathcal{K}$ and satisfying $(K 1)-(K 3)$.

The map $\psi: \mathbb{R}^{+} \rightarrow X$ is called a generalized trajectory of $\mathcal{R}$ if there exist a sequence of trajectories $\varphi_{m} \in \mathcal{R}$ and a sequence of points $0<t_{1}<t_{2}<\ldots<t_{m}<\ldots$ such that

$$
\psi(t)=\left\{\begin{array}{c}
\varphi_{1}(t) \text { if } 0 \leq t<t_{1} \\
\varphi_{2}\left(t-t_{1}\right) \text { if } t_{1} \leq t<t_{2} \\
\vdots \\
\varphi_{m}\left(t-t_{m-1}\right) \\
\text { if } t_{m-1} \leq t<t_{m} \\
\vdots
\end{array}\right.
$$

We note that a generalized trajectory is piecewise continuous, but not continuous in general. Let us denote the set of generalized trajectories by $\mathcal{R}_{g}$.

We shall define now an intermediate set of functions $\mathcal{R} \subset \mathcal{S} \subset \mathcal{K}$. Namely:

$$
\left.\mathcal{S}=\left\{\varphi \in \mathcal{K}: \exists \psi_{n} \in \mathcal{R}_{g} \text { such that } \sup _{t \in[0, T]} \rho\left(\psi_{n}(t), \varphi(t)\right) \rightarrow 0 \forall T>0\right)\right\}
$$

We notice that, although the maps $\psi_{n}$ can be discontinuous, the limit function $\varphi$ is continuous and, moreover, $\psi_{n}(t)$ converges to $\varphi(t)$ uniformly in every interval $[0, T]$.

Lemma 14 The set $\mathcal{S}$ satisfies properties $(K 1)-(K 3)$. The map $\bar{\Phi}: \mathbb{R}^{+} \times X \rightarrow \mathcal{P}(X)$ defined by

$$
y \in \bar{\Phi}(t, x) \text { if } \exists \varphi \in \mathcal{S} \text { such that } y=\varphi(t), \varphi(0)=x,
$$

is a strict multivalued semiflow.

Moreover, assume that the set $\mathcal{S}$ satisfies the following additional property:

$(K 4)$ For any sequence $\left\{\varphi^{n}\right\} \subset \mathcal{S}$ such that $\varphi^{n}(0) \rightarrow \varphi_{0}$ in $X$, there exists a subsequence $\varphi^{n_{k}}$ and $\varphi \in \mathcal{K}$ such that

$$
\varphi^{n_{k}} \rightarrow \varphi \text { in } C\left(\mathbb{R}^{+} ; X\right) .
$$

Then the map $(t, x) \mapsto \bar{\Phi}(t, x)$ has closed graph and is upper semicontinuous.

Proof. Properties $(K 1)-(K 2)$ are rather obvious.

Let us prove $(K 3)$. Let $\varphi_{1}, \varphi_{2} \in \mathcal{S}$ be such that $\varphi_{2}(0)=\varphi_{1}(s)$, where $s>0$. Then the function $\varphi(\cdot)$, defined by

$$
\varphi(t)=\left\{\begin{array}{l}
\varphi_{1}(t) \text { if } 0 \leq t \leq s, \\
\varphi_{2}(t-s) \text { if } s \leq t,
\end{array}\right.
$$

belongs to $\mathcal{K}$. We take the sequences $\psi_{n}^{j} \in \mathcal{R}_{g}, j=1,2$, such that $\psi_{n}^{j} \rightarrow \varphi^{j}$ uniformly in every interval $[0, T]$ and define the new sequence of generalized trajectories

$$
\psi_{n}(t)=\left\{\begin{array}{l}
\psi_{n}^{1}(t) \text { if } 0 \leq t<s, \\
\psi_{n}^{2}(t-s) \text { if } s \leq t .
\end{array}\right.
$$


We need to prove that $\psi^{n}$ converges to $\varphi$ uniformly in every interval $[0, T]$. If $T<s$, the result is obvious. Let $T \geq s$. We take an arbitrary sequence $t_{n} \rightarrow t_{0} \leq T$. We will show that $\psi_{n}\left(t_{n}\right) \rightarrow \varphi\left(t_{0}\right)$. If $t_{0} \neq s$, this is evident. Let $t_{n} \rightarrow s$. We split the sequence $t_{n}$ into two subsequences $t_{n_{j}}, t_{n_{k}}$ such that $\psi\left(t_{n_{j}}\right)=\psi_{n}^{1}\left(t_{n_{j}}\right)$ and $\psi\left(t_{n_{k}}\right)=\psi_{n}^{2}\left(t_{n_{k}}-s\right)$. Since $\psi_{n}^{1}\left(t_{n_{j}}\right) \rightarrow \varphi_{1}(s)=\varphi(s)$ and $\psi_{n}^{2}\left(t_{n_{k}}-s\right) \rightarrow \varphi_{2}(0)=\varphi(s)$, the result follows. As $\varphi(t)$ is a continuous function, the uniform convergence is proved. Hence, $\psi_{n} \rightarrow \varphi$ uniformly in every interval $[0, T]$ and $\varphi \in \mathcal{S}$.

Property (K3) implies that $\bar{\Phi}$ is a strict multivalued semiflow. Indeed, let $y \in \bar{\Phi}\left(t_{2}, \bar{\Phi}\left(t_{1}, x\right)\right)$. Then $y=\varphi_{2}\left(t_{2}\right)$ and $\varphi_{2}\left(t_{2}\right)=\varphi_{1}\left(t_{1}\right)$, where $\varphi_{1}, \varphi_{2} \in \mathcal{S}$ and $\varphi_{2}(0)=\varphi_{1}\left(t_{1}\right), \varphi_{1}(0)=x$. The concatenation of $\varphi_{1}, \varphi_{2}$, defined by (9) with $s=t_{1}$, belong to $\mathcal{S}$, and then $y=\varphi\left(t_{1}+t_{2}\right) \in \bar{\Phi}\left(t_{1}+t_{2}, x\right)$.

Finally, we shall prove that the map $(t, x) \mapsto \bar{\Phi}(t, x)$ has closed graph and is upper semicontinuous. Let $t_{n} \rightarrow t, x_{n} \rightarrow x$ and $y_{n} \rightarrow y$, where $y_{n} \in \bar{\Phi}\left(t_{n}, x_{n}\right)$. Then $y_{n}=\varphi_{n}\left(t_{n}\right)$, where $\varphi_{n} \in \mathcal{S}$. By (K4), up to a subsequence $\varphi_{n} \rightarrow \varphi \in \mathcal{K}$ in $C\left(\mathbb{R}^{+} ; X\right)$ and $\varphi(0)=x$. Also, by the definition of the set $\mathcal{S}$, we can choose $\psi_{n} \in \mathcal{R}_{g}$ such that

$$
\rho_{C}\left(\psi_{n}, \varphi_{n}\right)<\frac{1}{n}
$$

Here, we have used the metric of the space $C\left(\mathbb{R}^{+} ; X\right)$, no matter that $\psi_{n}$ are just piecewise continuous. Thus, for any $\epsilon>0$ there exists $N(\epsilon)$ such that

$$
\rho_{C}\left(\psi_{n}, \varphi\right) \leq \rho_{C}\left(\psi_{n}, \varphi_{n}\right)+\rho_{C}\left(\varphi_{n}, \varphi\right) \leq \frac{1}{n}+\frac{\epsilon}{2} \leq \epsilon \text { if } n \geq N
$$

It follows that $\psi_{n} \rightarrow \varphi$ uniformly in every interval $[0, T]$ and then $\varphi \in \mathcal{S}$. Hence, $y=\varphi(t) \in \bar{\Phi}(t, x)$. Therefore, $(t, x) \mapsto \bar{\Phi}(t, x)$ has closed graph.

Now, if we assume that the map is not upper semicontinuous, there would exist a neighborhood $O$ of $\bar{\Phi}(t, x)$ and sequences $t_{n} \rightarrow t, x_{n} \rightarrow x$ and $y_{n} \in \bar{\Phi}\left(t_{n}, x_{n}\right)$ such that $y_{n} \notin O$. Arguing in the same way as before we obtain that, up to a subsequence, $y_{n} \rightarrow y \in \bar{\Phi}(t, x)$, which is a contradiction.

Lemma 14 and Theorem 3 imply the following result.

Theorem 15 Assume that $\bar{\Phi}$ has a compact attracting set B. Let $(K 4)$ hold for the set $\mathcal{S}$. Then the strict multivalued semiflow $\bar{\Phi}$ possesses a global compact attractor $\mathcal{A}$, which is invariant.

\section{Applications}

This section is devoted to examples that illustrate the previous theoretical results. Namely, we deal with two problems where uniqueness of solutions is unknown or false, specifically, the three-dimensional Navier-Stokes equations and a reaction-diffusion problem. We observe that these equations generate non-strict semiflows.

\subsection{Application to the $3 D$ Navier-Stokes equations}

Let $\Omega$ be a bounded open subset of $\mathbb{R}^{3}$ with smooth enough boundary and, for a given $\nu>0$, consider the three dimensional Navier-Stokes system

$$
\left\{\begin{array}{l}
\frac{\partial u}{\partial t}-\nu \Delta u+(u \cdot \nabla) u=-\nabla p+f \\
\operatorname{div} u=0,\left.\quad u\right|_{\partial \Omega}=0, u(0)=u_{0} .
\end{array}\right.
$$


We define the usual function spaces

$$
\mathcal{V}=\left\{u \in\left(C_{0}^{\infty}(\Omega)\right)^{3}: \operatorname{div} u=0\right\}, \quad H=\overline{\mathcal{V}}^{\left(L^{2}(\Omega)\right)^{3}}, \quad V=\overline{\mathcal{V}}^{\left(H_{0}^{1}(\Omega)\right)^{3}},
$$

where $H$ and $V$ are separable Hilbert spaces (here $\bar{A}^{X}$ denotes closure of $A$ in the space $X$ ). We denote by $(\cdot, \cdot),\|\cdot\|$ and $((\cdot, \cdot)),\|\cdot\|_{V}:=\|\nabla \cdot\|$ the inner product and norm in $H$ and $V$, respectively. With an abuse of notation, $\|\cdot\|$ also stand for the norm in $\left(L^{2}(\Omega)\right)^{3}$. In addition, we identify $H$ and its dual $H^{*}$ and denote by $\langle\cdot, \cdot\rangle$ the pairing between $V$ and $V^{*}$. Finally, we write $H_{w}$ for the space $H$ endowed with the weak topology.

Let $A=-\mathbb{P} \Delta$ be the Stokes operator with Dirichlet boundary conditions, where $\mathbb{P}$ is the Helmholtz projection. We also consider the fractional power spaces

$$
V^{2 \alpha}=D\left(A^{\alpha}\right)=\left\{u \in H: \sum_{i=1}^{\infty} \lambda_{i}^{2 \alpha}\left|\left(u, e_{i}\right)\right|^{2}<\infty\right\}, \quad \alpha \geq 0,
$$

where $0<\lambda_{1} \leq \lambda_{2} \leq \cdots$ and $\left\{e_{1}, e_{2}, \cdots\right\}$ are the eigenvalues and the eigenfunctions of $A$, respectively. Note that $V^{0}=H$ and $V^{1}=V$. In addition, we denote by $V^{-2 \alpha}$ the dual space of $V^{2 \alpha}$ and the norms in $V^{2 \alpha}$ and $V^{-2 \alpha}$ will be denoted by $\|\cdot\|_{V^{2 \alpha}}$ and $\|\cdot\|_{V^{-2 \alpha}}$, respectively, while $\langle\cdot, \cdot\rangle_{2 \alpha}$ will denote the pairing between $V^{-2 \alpha}$ and $V^{2 \alpha}$. It is well known that $V^{\alpha} \subset V^{\beta}$ for $\alpha>\beta$ with a dense and compact embedding, while $V^{2 \alpha} \subset\left(H^{2 \alpha}(\Omega)\right)^{3}$ with a continuous embedding.

For $u, v, w \in V$ we write

$$
b(u, v, w):=\int_{\Omega} \sum_{i, j=1}^{3} u_{i} \frac{\partial v_{j}}{\partial x_{i}} w_{j} d x,
$$

where $b$ is a trilinear continuous form on $V$ and $b(u, v, v)=0$ if $u \in V$ and $v \in\left(H_{0}^{1}(\Omega)\right)^{3}$. As usual, for $u, v \in V$ we denote by $B(u, v)$ the element of $V^{*}$ defined by $\langle B(u, v), w\rangle=b(u, v, w)$ for all $w \in V$.

Finally, we assume that $f \in H$. Then it is well known [20] that for any $u_{0} \in H$ there exists at least one globally defined weak solution $u(\cdot)$ of (10) satisfying the energy inequality

$$
V(u(t)) \leq V(u(s)) \quad \text { for all } t \geq s \text { for a.a. } s>0 \text { and for } s=0,
$$

where

$$
V(u(t)):=\frac{1}{2}\|u(t)\|^{2}+\nu \int_{0}^{t}\|u\|_{V}^{2} d \tau-\int_{0}^{t}(f, u(\tau)) d \tau .
$$

In fact, every globally defined weak solution satisfies $\left.u \in C[0, T], H_{w}\right)$ for all $T>0$.

For every weak solution satisfying (11) we have

$$
\|u(t)\|^{2} \leq e^{-\nu \lambda_{1}(t-s)}\left(\|u(s)\|^{2}-\frac{1}{\nu^{2} \lambda_{1}^{2}}\|f\|^{2}\right)+\frac{1}{\nu^{2} \lambda_{1}^{2}}\|f\|^{2},
$$

for all $t \geq s$, a.a. $s>0$ and $s=0$. This is proved in [5, Proposition 7.3] (see also [9, Lemma 5.20]).

We observe that inequality (12) allows to obtain a bounded absorbing set for all the solutions satisfying it. However, such a choice of solutions has a problem. If we define the translation $u_{\tau}(t)=u(t+\tau)$, then property (11) fails for $u(\cdot)$ at $s=0$. Thus, property $(K 2)$ is not true. Also, for the associated multivalued map $G(t, \cdot)$ the key property $G(t+s, x) \subset G(t, G(s, x))$ fails (see [4, Section 4.3.1] for more details).

Due to this problem we consider all weak solutions satisfying the weaker energy inequality

$$
V(u(t)) \leq V(u(s)) \text { for all } t \geq s \text { a.a. } s>0 .
$$


Lemma 16 Every weak solution such that (13) holds satisfies

$$
F(u(t)) \leq F(u(s)) \quad \text { for all } t \geq s \text { and a.a. } s>0,
$$

where

$$
F(u(t))=\left(\|u(t)\|^{2}-R_{0}^{2}\right) e^{\delta t}, \quad \delta=\lambda_{1} \nu, R_{0}^{2}=\frac{1}{\nu^{2} \lambda_{1}^{2}}\|f\|^{2} .
$$

If (13) is satisfied also for $s=0$, then (14) holds also at $s=0$.

Proof. First, note that (14) is satisfied for the same $s$ as (13). Choose an arbitrary $s>0$ for which (13) holds and define $\bar{u}(\tau):=u(\tau+\cdot)$ for $\tau \geq 0$. Since $\bar{u}(\cdot)$ is a weak solution satisfying (11), it follows from (12) that

$$
F(u(\tau+s))=F(\bar{u}(\tau)) \leq F(\bar{u}(0))=F(u(s)) \quad \text { for all } \tau \geq 0 .
$$

The second part is a direct consequence of (12).

Define $B_{R}=\{u \in H:\|u\| \leq R\}$ and for any $R \geq R_{0}$ define the multivalued map $\Phi_{R}: \mathbb{R}^{+} \times B_{R} \rightarrow$ $\mathcal{P}\left(B_{R}\right)$ by

$$
\Phi_{R}\left(t, u_{0}\right):=\left\{\begin{array}{c}
u(t): u(\cdot) \text { is a globally defined weak solution with } u(0)=u_{0} \\
\text { such that (13) holds and }\|u(r)\| \leq R \text { for all } r \geq 0
\end{array}\right\} .
$$

In view of (12) there exists at least one weak solution such that the estimate $F(u(t)) \leq F(u(s))$ holds for all $t \geq s$ for a.a. $s \in(0, t)$ and for $s=0$. Thus, for $\left\|u_{0}\right\| \leq R$ we have

$$
\|u(r)\|^{2} \leq e^{-\delta r}\left(\left\|u_{0}\right\|^{2}-R_{0}^{2}\right)+R_{0}^{2} \leq R^{2} \text { for all } r \geq 0,
$$

which means that the map $\Phi_{R}$ has non-empty values and is correctly defined.

We consider then the phase space $X_{R}=B_{R}$ endowed with the induced weak topology of the space $H_{w}$. This space is a compact metrizable space. Thus it is obvious that $\Phi_{R}$ possesses a compact, positively semi-invariant absorbing set. It is shown in [9, Theorem 5.32] (see also [12]) that the map $\Phi_{R}$ is a multivalued semiflow satisfying Assumptions A1-A2 and that it has a global compact attractor $\mathcal{A}_{R}^{(0)}$. It is also proved there that $\mathcal{A}_{R}^{(0)}=\mathcal{A}_{R_{0}}^{(0)}$ for all $R \geq R_{0}$.

We are, however, not able to prove the inclusion $\Phi_{R}\left(t, \Phi_{R}\left(r, u_{0}\right)\right) \subset \Phi_{R}\left(t+r, u_{0}\right)$. The reason is that the concatenation of two solutions satisfying (13), i.e.,

$$
\theta(p)=\left\{\begin{array}{l}
u(p), p \in[0, r] \\
v(p), p \in[r, t+r]
\end{array}\right.
$$

need not satisfy condition (13). Hence, we have, in general, a non-strict multivalued semiflow.

Let $\mathcal{R}$ be the set of all weak solutions as given in (15). Then conditions $(K 1)-(K 2)$ hold for it and $\mathcal{R}$ generates the multivalued semiflow $\Phi_{R}$, so that (7) holds.

We can then define the multivalued semiflows $\bar{\Phi}_{R}^{(n)}: X_{R} \rightarrow \mathcal{P}\left(X_{R}\right)$ given in Section 3, which are strict at the $n$-dyadics $\widehat{\mathfrak{D}}_{n}$. It follows from the results in that section that $\bar{\Phi}_{R}^{(n)}$ has the global compact attractor $\mathcal{A}_{R}^{(n)}$ (which are strictly invariant at the $n$-dyadics $\widehat{\mathfrak{D}}_{n}$ ) and that

$$
\mathcal{A}_{R}^{(0)} \subset \mathcal{A}_{R}^{(1)} \subset \cdots \subset \mathcal{A}_{R}^{(n)} \subset \cdots
$$

Also, $\mathcal{A}_{R}^{(\infty)}=\cup_{n} \mathcal{A}_{R}^{(n)}$ is strictly invariant for the multivalued semiflow $\Phi_{R}^{(\infty)}=\cup_{n} \bar{\Phi}_{R}^{(n)}$ at the dyadics $\widehat{\mathfrak{D}}$ $=\cup_{n} \widehat{\mathfrak{D}}_{n}$. However, $\mathcal{A}_{R}^{(\infty)}$ is not an attractor for $\Phi_{R}^{(\infty)}$, but $\widehat{\mathcal{A}}:=\mathcal{A}_{R}^{(0)} \times \mathcal{A}_{R}^{(1)} \times \cdots \times \mathcal{A}_{R}^{(n)} \times \cdots$ is a strictly invariant attractor for the time-one map $\widehat{\Phi}_{R}^{(\infty)}: \widehat{X}_{R} \rightarrow \mathcal{P}\left(\widehat{X}_{R}\right)$, where $\widehat{X}_{R}:=\prod_{i=0}^{\infty} X_{R}$, given by

$$
\widehat{\Phi}^{(\infty)}(\hat{x}):=\left(\Phi^{(0)}\left(1, x_{0}\right), \Phi^{(1)}\left(1, x_{1}\right), \ldots, \Phi^{(n)}\left(1, x_{n}\right), \cdots,\right),
$$


where $\hat{x}:=\left(x_{0}, x_{1}, \ldots, x_{n}, \cdots\right)$.

In addition, we consider the multivalued semiflow $\bar{\Phi}_{R}^{(\infty)}: X_{R} \rightarrow \mathcal{P}\left(X_{R}\right)$ as defined in Section 3.6.

Theorem 17 For each $R \geq R_{0}$ the multivalued semiflow $\bar{\Phi}_{R}^{(\infty)}$ has a compact global attractor $\mathcal{A}_{R}$, which is weakly positively semi-invariant.

Proof. Since $\bar{\Phi}^{(\infty)}$ has a compact absorbing set and (7) holds, in order to apply Theorem 13 we need to check only that condition Assumption A3 is satisfied.

Consider a sequence of pseudo-trajectories $\psi_{n}(\cdot)$ with $\psi(0)=x_{n} \in X_{R}$ and $x_{n} \rightarrow x$ in $X_{R}$. Then

$$
\psi_{n}(t)=u_{m}^{n}\left(t-t_{m-1}^{n}\right) \quad \text { if } t_{m-1}^{n} \leq t \leq t_{m}^{n}, \quad m \in \mathbb{N},
$$

where the $u_{j}^{n}(\cdot)$ are globally defined weak solutions of (10) satisfying the properties given in (15). For any $T>0$ this sequence is bounded in $L^{\infty}(0, T ; H)$, which is obvious as $u_{j}^{n}(t) \in X_{R}$ for all $t \geq 0$, so $\left\|u_{j}^{n}(t)\right\| \leq R$. Hence, for some subsequence,

$$
\psi_{n} \rightarrow \psi \text { weakly star in } L^{\infty}(0, T ; H) .
$$

We can derive a uniform estimate of $\frac{d \psi_{n}}{d t}$ in the space $L^{\infty}\left(0, T ; V^{-3}\right)$. First note that

$$
\begin{aligned}
\left\|A u_{m}^{n}(\tau)\right\|_{V^{-2}} & =\sup _{\|w\|_{2} \leq 1}\left|\left\langle A u_{m}^{n}(\tau), w\right\rangle_{2}\right|=\sup _{\|w\|_{2} \leq 1}\left|\left(u_{m}^{n}(\tau), A w\right)\right| \\
& \leq\left\|u_{m}^{n}(\tau)\right\| \leq R \quad \text { for all } \tau \in\left[0, t_{m}^{n}-t_{m-1}^{n}\right] .
\end{aligned}
$$

On the other hand, for $u \in V$ we obtain

$$
\begin{aligned}
\|B(u, u)\|_{V^{-3}} & =\sup _{\|w\|_{3} \leq 1}|b(u, u, w)|=\sup _{\|w\|_{3} \leq 1}|b(u, w, u)| \\
& \leq \sup _{\|w\|_{3} \leq 1}\left|\int_{\Omega} \sum_{i, j=1}^{3} u_{i} \frac{\partial w_{j}}{\partial x_{i}} u_{j} d x\right| .
\end{aligned}
$$

Since $w \in V^{3} \subset\left(H^{3}(\Omega)\right)^{3}$, we have $\frac{\partial w_{j}}{\partial x_{i}} \in H^{2}(\Omega)$. Thus by the continuity of the embedding $H^{2}(\Omega) \subset$ $L^{\infty}(\Omega)$, we have

$$
\int_{\Omega}\left|u_{i} \frac{\partial w_{j}}{\partial x_{i}} u_{j}\right| d x \leq\left\|\frac{\partial w_{j}}{\partial x_{i}}\right\|_{L^{\infty}}\left\|u_{i}\right\|_{L^{2}}\left\|u_{j}\right\|_{L^{2}},
$$

so there exists a constant $K>0$ such that $\|B(u, u)\|_{V^{-3}} \leq K\|u\|^{2}$. Hence, for a.a. $\tau \in\left(0, t_{m}^{n}-t_{m-1}^{n}\right)$, we have

$$
\left\|B\left(u_{m}^{n}(\tau), u_{m}^{n}(\tau)\right)\right\|_{V^{-3}} \leq K R^{2} .
$$

The equality

$$
\frac{d u_{m}^{n}}{d t}=-\nu A u_{m}^{n}-B\left(u_{m}^{n}, u_{m}^{u}\right)+f
$$

in $V^{*}$, the inequalities (16), (17) and the continuous embeddings $H \subset V^{-2} \subset V^{-3}$ imply that

$$
\left\|\frac{d u_{m}^{n}(\tau)}{d t}\right\|_{V^{-3}} \leq \nu C_{1} R+K R^{2}+C_{2}\|f\| \text { for a.a. } \tau \in\left(0, t_{m}^{n}-t_{m-1}^{n}\right) .
$$

Since $\psi_{n}$ are absolutely continuous on $[0, T]$ with values in $V^{-3}$, it follows that $\frac{d \psi_{n}}{d t}$ is bounded in the space $L^{\infty}\left(0, T ; V^{-3}\right)$ for any $T>0$. 
Thus, the functions $\psi_{n}$ with values in the space $V^{-3}$ are equicontinuous. On the other hand, the compact embedding $H \subset V^{-3}$ implies that $\psi_{n}(t)$ is relatively compact in $V^{-3}$ for any $t$. Then the AscoliArzelà theorem implies that $\psi_{n}(\cdot) \rightarrow \psi(\cdot)$ in $C\left([0, T], V^{-3}\right)$. Since $\psi_{n}\left(t_{n}\right)$ is bounded in $H$, a standard contradiction argument implies that for any $t_{n} \rightarrow t_{0}$ with $t_{n}, t_{0} \in[0, T]$, we have

$$
\psi_{n}\left(t_{n}\right) \rightarrow \psi\left(t_{0}\right) \quad \text { weakly in } H \text {. }
$$

By a diagonal argument we obtain a subsequence such that these convergences are true for all $T>0$.

Also, since $\psi \in L^{\infty}(0, T ; H)$ and $\psi \in C\left([0, T], V^{-3}\right)$, Lemma 1.4 in [20, p.262] implies that $\psi(\cdot) \in$ $C\left([0, T], H_{w}\right)$, for any $T>0$. Therefore, Assumption A3 holds.

Theorem 13 implies then that the multivalued semiflow $\bar{\Phi}_{R}^{(\infty)}$ has a compact global attractor $\mathcal{A}_{R}$, which is weakly positively semi-invariant (as well as being negatively semi-invariant).

\subsection{Application to a reaction-diffusion problem}

In this section we consider a reaction-diffusion problem where uniqueness of solution is unknown, and the results above can be applied.

More exactly, consider the following problem (see [8] for a more involved description of the problem). Let be given a bounded domain $\Omega \subset \mathbb{R}^{3}$ with smooth enough boundary $\partial \Omega$, functions $h \in L^{2}(\Omega)$, $f \in C(\mathbb{R})$, and positive constants $C_{1}, C_{2}, \alpha$ such that

$$
|f(s)| \leq C_{1}\left(1+|s|^{3}\right) \quad \text { and } \quad f(s) s \geq \alpha|s|^{4}-C_{2},
$$

for all $s \in \mathbb{R}$. The norm in $L^{2}(\Omega)$ wil be denoted by $\|\cdot\|$.

Now, we consider the problem

$$
\left\{\begin{array}{l}
u_{t}-\Delta u+f(u)=h, \quad x \in \Omega, t>0 \\
\left.u\right|_{\partial \Omega}=0
\end{array}\right.
$$

Definition 18 A function $u \in L_{l o c}^{2}\left(0,+\infty ; H_{0}^{1}(\Omega)\right) \cap L_{l o c}^{4}\left(0,+\infty ; L^{4}(\Omega)\right)$ is called a weak solution to (20) on $(0,+\infty)$ if it satisfies the equation in $\mathcal{D}^{\prime}\left(0,+\infty ; H^{-1}(\Omega)\right)$, i.e., for all $T>0, v \in H_{0}^{1}(\Omega)$, and $\eta \in C_{0}^{\infty}(0, T)$ it holds

$$
-\int_{0}^{T}(u, v) \eta_{t} d t+\int_{0}^{T}\left[(u, v)_{H_{0}^{1}(\Omega)}+(f(u), v)-(h, v)\right] \eta d t=0,
$$

where we are denoting by $(\cdot, \cdot)$ the scalar product in $L^{2}$ or the duality action between $L^{p}$ and its conjugate $L^{q}$, and $(\cdot, \cdot)_{H_{0}^{1}(\Omega)}=(\nabla \cdot, \nabla \cdot)$.

It is well known (e.g. cf. [6, page 284] or [1, Theorem 2]) that a weak solution must also satisfy $u \in C\left([0,+\infty) ; L^{2}(\Omega)\right.$ ), and that for any $u_{0} \in L^{2}(\Omega)$ there exists at least one weak solution (possibly non-unique) to (20) with $u(0)=u_{0}$.

According to the previous theoretical results given in Section 4, we will denote

$$
\mathcal{K}=\{u(\cdot): u(\cdot) \text { is a weak solution to }(20)\} .
$$

It is not difficult to see that $\mathcal{K}$ satisfies (K1)-(K3) (see [22, Lemma 5.8]). Let us recall the following results. 
Lemma 19 [9, Theorem 3.11, page 49] Let $\left\{u^{n}\right\}$ be a sequence of weak solutions of (20), and $u^{n}(0) \rightarrow u_{0}$ weakly in $L^{2}(\Omega)$. Then there exists a subsequence (denoted again by $u^{n}$ ), and a weak solution of (20) $u$ satisfying $u(0)=u_{0}$, such that $u^{n}\left(t_{n}\right) \rightarrow u\left(t_{0}\right)$ weakly in $L^{2}(\Omega)$ for any sequence of times $\left\{t_{n}\right\} \subset[0, T]$ with $t_{n} \rightarrow t_{0} \in[0, T]$. Also, if $t_{0} \in(0, T)$, then $u^{n}\left(t_{n}\right) \rightarrow u\left(t_{0}\right)$ strongly in $L^{2}(\Omega)$.

Moreover, if $u^{n}(0) \rightarrow u_{0}$ strongly in $L^{2}(\Omega)$, then $u^{n}\left(t_{n}\right) \rightarrow u_{0}$ strongly in $L^{2}(\Omega)$ for $t_{n} \searrow 0$.

Theorem 20 [11, Theorem 10] (see also [9] or [22]) The set $\mathcal{K}$ generates a strict multivalued semiflow $G: \mathbb{R}^{+} \times L^{2}(\Omega) \rightarrow P\left(L^{2}(\Omega)\right)$, which possesses a global compact invariant attractor $\mathcal{A}_{\mathcal{K}}$.

However, following [8, Section 5], in which the behaviour of more regular solutions is described, we may consider the following concept.

Definition 21 A function $u \in L_{\text {loc }}^{2}\left(0,+\infty ; H_{0}^{1}(\Omega)\right) \cap L_{\text {loc }}^{4}\left(0,+\infty ; L^{4}(\Omega)\right)$ is called a regular solution to (20) on $(0,+\infty)$ if it is a weak solution that additionally satisfies

$$
u \in L^{\infty}\left(\varepsilon, T ; H_{0}^{1}(\Omega)\right), \quad u_{t} \in L^{2}\left(\varepsilon, T ; L^{2}(\Omega)\right) \quad \forall 0<\varepsilon<T .
$$

Observe that if $u$ is a regular solution to (20), then, from (19), $f(u) \in L^{2}((\varepsilon, T) \times \Omega)$ and $u \in$ $L^{2}(\varepsilon, T ; D(-\Delta))$ for all $0<\varepsilon<T$, and so $u \in C\left((0,+\infty) ; H_{0}^{1}(\Omega)\right)$.

Actually, the existence of regular solutions to (20), even with initial datum $u_{0} \in L^{2}(\Omega)$, is guaranteed by $[8$, Theorem 22].

Let us denote

$$
\mathcal{R}=\{u(\cdot): u(\cdot) \text { is a regular solution to }(20)\} .
$$

It is not difficult to see that $\mathcal{R}$ satisfies (K1)-(K2). However, due to (21), (K3) does not hold. It is proved in [8] that $\mathcal{R}$ generates a multivalued semiflow $\widetilde{\Phi}$ having a global attractor $\mathcal{A}_{\mathcal{R}}$, which is compact in $H_{0}^{1}(\Omega)$ (but it is not proved to be invariant).

Following the notation introduced in Section 4 , we consider the set of generalized trajectories $\mathcal{R}_{g}$, and

$$
\mathcal{S}=\left\{\psi \in \mathcal{K}: \exists\left\{\psi_{n}\right\} \subset \mathcal{R}_{g} \text { such that } \sup _{t \in[0, T]}\left\|\psi_{n}(t)-\varphi(t)\right\| \rightarrow 0 \forall T>0\right\} .
$$

Roughly speaking, $\mathcal{S}$ is the set of weak solutions that can be approximated by a generalized concatenation of regular ones.

Theorem 22 Under the above conditions, $\mathcal{S}$ satisfies assumptions (K1)-(K4). In particular, the map $\bar{\Phi}:[0,+\infty) \times L^{2}(\Omega) \rightarrow \mathcal{P}\left(L^{2}(\Omega)\right)$, defined by

$$
\bar{\Phi}(t, x)=\{y=\varphi(t): \varphi \in \mathcal{S}, \varphi(0)=x\},
$$

is a strict multivalued semiflow.

Moreover, it possesses a global compact invariant attractor $\mathcal{A}$. Moreover, $\mathcal{A}_{\mathcal{R}} \subset \mathcal{A} \subset \mathcal{A}_{\mathcal{K}}$.

Proof. It follows immediately from Lemma 19 (note that the weak solutions $u$ are continuous) that (K4) holds. In fact, in this case the property is stronger than (K4), as the convergence is true for every sequence in $\mathcal{K}$, and not only in $\mathcal{S}$.

The fact that $\bar{\Phi}$ is a strict semiflow is an immediate consequence of (K1)-(K3) and Lemma 14. Also, by (K4) and Lemma 14 again we obtain that the map $(t, x) \mapsto \bar{\Phi}(t, x)$ has closed graph and is upper semicontinuous.

On the other hand, in view of Theorem 20 and the obvious inclusion $\bar{\Phi}(t, x) \subset G(t, x)$ we have

$$
\operatorname{dist}\left(\bar{\Phi}(t, B), \mathcal{A}_{\mathcal{K}}\right) \leq \operatorname{dist}\left(G(t, B), \mathcal{A}_{\mathcal{K}}\right) \rightarrow 0, \text { as } t \rightarrow+\infty,
$$


for every bounded set $B$. Hence, $\mathcal{A}_{\mathcal{K}}$ is a compact attracting set for $\bar{\Phi}$.

Therefore, the existence and properties of the global attractor follows from Theorem 3 . The inclusion $\mathcal{A}_{\mathcal{R}} \subset \mathcal{A} \subset \mathcal{A}_{\mathcal{K}}$ follows from $\widetilde{\Phi} \subset \bar{\Phi} \subset \Phi$.

\section{Acknowledgments}

Partially supported by spanish Ministerio de Ciencia e Innovación and FEDER, projects MTM201122411 and MTM2012-31698, the Consejería de Innovación, Ciencia y Empresa (Junta de Andalucía) under the Proyecto de Excelencia P07-FQM-02468 and the Consejería de Cultura y Educación (Comunidad Autónoma de Murcia), grant 08667/PI/08.

We thank the anonymous referee for his/her useful comments.

\section{References}

[1] Anguiano, M., Caraballo, T., Real, J., Valero, J.: Pullback attractors for reaction-diffusion equations in some unbounded domains with an $H^{-1}$-valued non-autonomous forcing term and without uniqueness of solutions. Discrete Contin. Dyn. Syst. Ser. B, 14, 307-326 (2010)

[2] Aubin, J.P., Cellina, A.: Differential Inclusions, Set-Valued Maps and Viability Theory. SpringerVerlag, Berlin (1984)

[3] Aubin, J.P., Frankowska, H.: Set-Valued Analysis. Birkhäuser, Basel (1990)

[4] Balibrea, F., Caraballo, T., Kloeden, P.E., Valero, J.: Recent developments in dynamical systems: three perspectives. Internat. J. Bifur. Chaos, 20, 2591-1636 (2010)

[5] Ball, J.M.: Continuity properties and global attractors of generalized semiflows and the NavierStokes equations, in Mechanics: from Theory to Computation. Springer-Verlag, New York, 447-474 (2000)

[6] Chepyzhov, V.V., Vishik, M.I.: Attractors for Equations of Mathematical Physics. American Mathematical Society, Providence, RI (2002)

[7] Filippov, A.F.: Classical solutions of differential equations with multi-valued right-hand sides. SIAM J. Control Optim. 5, 609-621 (1967)

[8] Kapustyan, O.V., Kasyanov, P.O., Valero, J.: Structure and regularity of the global attractor of a reaction-diffusion equation with non-smooth nonlinear term. Preprint Arxiv-1209.2010v1 (2012)

[9] Kapustyan, A.V., Melnik, V.S., Valero, J., Yasinsky, V.V.: Global Attractors of Multi-valued Dynamical Systems and Evolution Equations without Uniqueness. Naukova Dumka, Kyiv (2008)

[10] Kapustyan, A.V., Valero, J.: Attractors of multivalued semiflows generated by differential inclusions and their approximations. Abstr. Appl. Anal. 5, 33-46 (2000)

[11] Kapustyan, A.V., Valero, J.: On the connectedness and asymptotic behaviour of solutions of reactiondiffusion systems. J. Math. Anal. Appl. 323, 614-633 (2006)

[12] Kapustyan, A.V., Valero, J.: Weak and strong attractors for the 3D Navier-Stokes system. J. Differential Equations, 240, 249-278 (2007) 
[13] Kloeden, P.E., Marín-Rubio, P.: Negatively invariant sets and entire trajectories of set-valued dynamical systems. Set-Valued Var. Anal. 19, 43-57 (2011)

[14] Melnik, V.S., Valero, J.: On attractors of multivalued semi-flows and differential inclusions. SetValued Anal. 6, 83-111 (1998)

[15] Melnik, V.S., Valero, J.: Addendum to "On attractors of multivalued semiflows and differential inclusions". Set-Valued Anal. 16, 507-509 (2008)

[16] Robinson, J.: Infinite-dimensional Dynamical Systems. Cambridge University Press, Cambridge (2001)

[17] Roxin, E.: Stability in general control systems. J. Differential Equations, 1, 115-150 (1965)

[18] Smirnov, G.V.: Introduction to the Theory of Differential Inclusions. Amer. Math. Soc., Providence (2002)

[19] Szegö, G.P., Treccani, G.: Semigruppi di Trasformazioni Multivoche. Springer Lecture Notes in Mathematics, vol. 101, Springer-Verlag, Heidelberg (1969)

[20] Temam, R.: Navier-Stokes Equations. North-Holland, Amsterdam (1979)

[21] Tolstonogov, A.A.: On solutions of evolution inclusions I, Sibirsky Math. Zh. 33, 161-174 (1992). (English translation in Siberian Math. J. 33 (1992))

[22] Zgurovsky, M.Z., Kasyanov, P.O., Kapustyan, O.V., Valero, J., Zadoianchuk, N.V.: Evolution Inclusions and Variational Inequalities for Earth Data Processing III. Springer-Verlag, Berlin (2012). 